\title{
Modelo de registro fotogramétrico 2D y 3D del patrimonio edificado de Cuenca
}

\author{
2D and 3D Photogrammetric Registration Model \\ of the Built Heritage of Cuenca
}

\author{
Paúl Ochoa-Arias \\ Universidad del Azuay \\ pochoa@uazuay.edu.ec \\ https://orcid.org/0000-0002-5791-3041 \\ Omar Andrés Delgado-Pinos \\ Universidad del Azuay \\ adelgado@uazuay.edu.ec \\ https://orcid.org/0000-0003-4114-3481
}

\begin{abstract}
Resumen
La gestión participativa del patrimonio cultural supone la colaboración de diferentes propuestas y actores. En ese sentido, se ha desarrollado la iniciativa descrita en el presente artículo, la misma que permite por medio de fotogrametría, registrar gráficamente el patrimonio edificado relevante del centro histórico de la ciudad de Cuenca y gestionarlo en un Sistema de Información Geográfica. Usando cámaras fotográficas individuales o montadas en drones, se procede el levantamiento fotográfico del inmueble u objeto (desde múltiples ángulos), a este material se le aplican ciertos procesos, apoyados en software especializado, para obtener un mosaico de imágenes con el cual se puede conseguir la corrección de la perspectiva en las imágenes (proceso conocido como fotogrametría) con lo que se obtienen productos digitales imprimibles en formas bidimensionales (papel) y tridimensionales (resinas) con escala y precisión. Comparativamente con otros procedimientos, tiene varias ventajas: facilidad, rapidez, costos bajos, calidad, aunque evidencia ciertas limitaciones. Luego de la elaboración del proyecto, se puede establecer que las disponibilidades técnicas y metodológicas permiten conseguir un modelo de información documentada digitalmente e integrada en un Sistema de Información Geográfica; el cual dispone de información predial (ficha catastral), delimitación de las áreas de valor patrimonial, representación de las edificaciones bidimensional y tridimensionalmente, acceso al respectivo sitio web, y un potencial amplio para la catalogación, clasificación, mantenimiento, recuperación, estudio y promoción del patrimonio edificado.
\end{abstract}

\section{Palabras clave}

Fotogrametría arquitectural, patrimonio edificado, modelos 3D, ortofoto, Sistemas de Información Geográfica, Geomática.

Forma sugerida de citar: Ochoa-Arias, P., \& Delgado-Pinos O.A. (2020). Modelo de registro fotogramétrico 2D y 3D del patrimonio edificado de Cuenca. Univeritas, 33, pp. 163-180. 


\begin{abstract}
Participatory cultural heritage management involves collaboration of different proposals and participants. In this article, the initiative that allows to register graphically the relevant built heritage of the historic center of the city of Cuenca by means of photogrammetry, and the management of it in a Geographic information System have been developed. The photographic study of the property or object is carried out (from multiple angles) by using individual or mounted on drone's cameras. Certain processes, supported by specialized software, are applied to this material to obtain a mosaic of images with which it is possible to obtain the correction of the perspective in the images (photogrammetry). Printable digital products are obtained in two-dimensions (paper) and three-dimensions (resins) forms with scale and precision. Compared to other procedures, this proposal has several advantages: ease, speed, low costs, good quality of the products. Although, we must also acknowledge some limitations. After the development of the project, it can be established that the technical and methodological availability allows to obtain a digitally documented information model integrated into a Geographic Information System; which has property information (cadastral record), delimitation of the areas of heritage value, representation of the two-dimensional and three-dimensional buildings, access to the respective website.
\end{abstract}

Keywords

Architectural photogrammetry, built heritage, 3D models, orthophotography, Geographic Informatio System, Geomatics.

\title{
Introducción
}

El paisaje entendido como: "La pauta de distribución de los elementos significativos en la organización del espacio" (Pisón, 1998), plantea de inicio el condicionamiento que el medio natural establece en la estructuración del medio edificado, el mismo que se evidencia en la manera singular en la que se configuran una ciudad.

Adicionalmente y de manera paulatina, se observa en la arquitectura de una ciudad, la influencia proveniente de sucesos culturales, económicos y sociales a lo largo del devenir histórico de los pueblos (Roura \& Ochoa, 2014).

En efecto, la configuración de una ciudad es el producto de la concurrencia de diversos elementos que la caracterizan. En el caso de la ciudad de Cuenca, su centro histórico ha recibido el influjo de algunos estilos ar- 
quitectónicos como: Neoclásico Francés, Árabe Español, Art Nouveau, Art Déco, entre otros. Lo cual ha constituido una serie de tramos urbanos de mayor y menor relevancia estética en función de la concordancia que adquiere el conjunto.

La influencia de estas corrientes se produce en la arquitectura de la ciudad a inicios del siglo XX, pues el excedente económico obtenido por una élite de cuencanos, a partir de la comercialización de la cascarilla, el sombrero de paja toquilla y la producción minera; les permitió viajar y adquirir costumbres, modas y demás referentes provenientes sobre todo de Europa, lo cual se materializa en un paisaje de características urbanas que lo alejaba de su origen silencioso, casi rural, heredado de la época colonial.

Es así como Carlos Jaramillo (Universidad de Cuenca / GAD Municipal del Cantón Cuenca, 2017) lo refiere:

... no es un cuadro renacentista que debe ser visto desde una sola perspectiva. Más bien, es un collage espacial compuesto de reliquias arquitectónicas, historias yuxtapuestas, tiempos amontonados, textos rotos, fragmentos, cronotopos, fenotopos... donde sus relaciones son móviles y forman, por eso, un conjunto simbólico de alta significación...

La singularidad del centro histórico de la ciudad de Cuenca es puesta a consideración de la UNESCO, con el propósito de solicitar su inclusión en el listado de patrimonio cultural de la humanidad, la misma que se consigue el 4 de diciembre de 1999.

La declaratoria (expediente 863) reconoce entre otros aspectos “... la belleza de su centro histórico, con un tipo particular de arquitectura republicana ..." y "...la armonía que guarda con su entorno geográfico..." (UNESCO, 2019).

En el expediente se establece la delimitación de áreas de valor patrimonial en 4 áreas: primer orden, de respeto, especial y arqueológica (Figura 1).

El mencionado expediente señala que se tienen las siguientes edificaciones en el área definida en 1994:

- Edificaciones de valor Monumental 26

- Edificaciones de Valor Arquitectónico I 179

- Edificaciones de Valor Arquitectónico II 423

- Edificaciones de Valor Ambiental 830

- Total 1458 


\section{Figura 1 \\ Categorización del centro histórico de la ciudad de Cuenca}

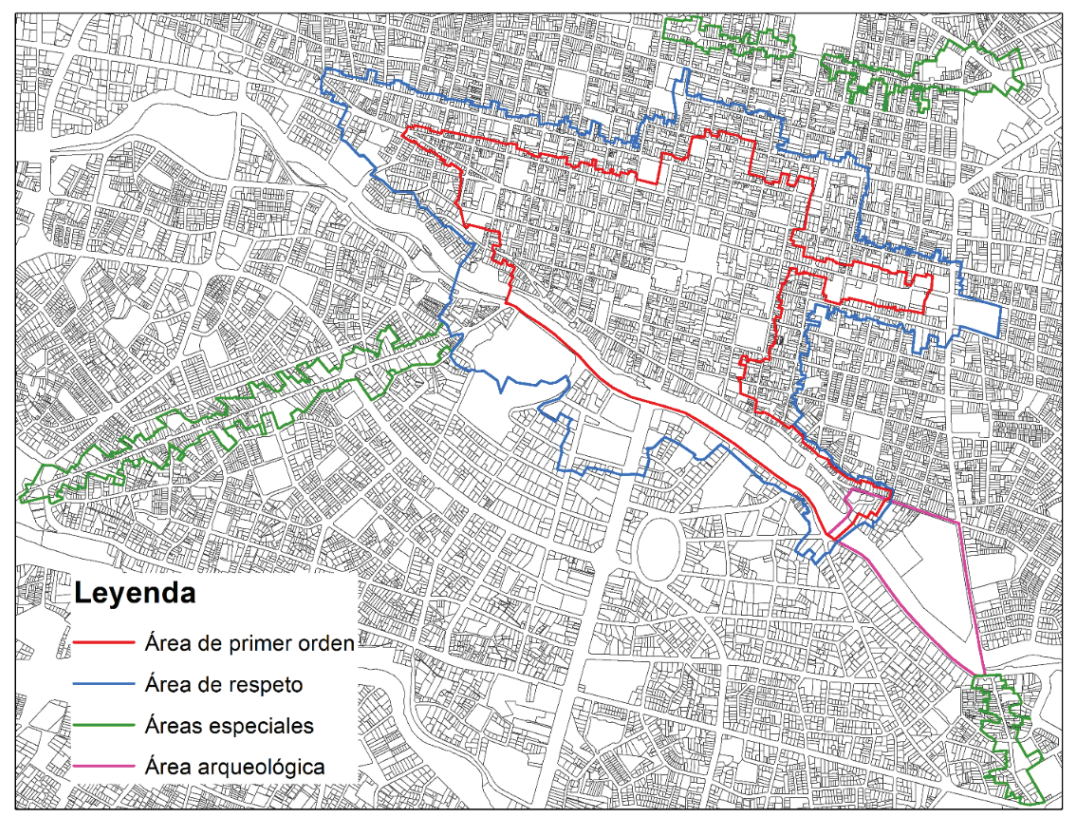

Fuente: Elaboración propia a partir de información del GAD Municipal de Cuenca del año 1998

Consecuente con la necesidad de disponer de documentación gráfica y temática que permita dar soporte a una adecuada gestión del patrimonio edificado de la ciudad, la Universidad del Azuay desde el año 2009, por medio de la línea de investigación de Geomática y Territorio, ha desarrollado un trabajo que integra: el registro de las edificaciones patrimoniales por medio de un Sistema de Información Geográfica, la modelación gráfica bidimensional y tridimensional de las edificaciones utilizando la fotogrametría.

El desarrollo alcanzado considera lo que algunos autores como Mario Santana (Santana, 2013) describe como los retos de la digitalización de sitios de patrimonio, y se indica que se debe tener cuidado en que los repositorios sean compartidos, contengan información fiable y que sus registros sean almacenados cuidadosamente para evitar su pérdida (a ellos les denomina: fragmentación, fiabilidad y longevidad respectivamente). 
El acercamiento a la tecnología fotogramétrica se inicia con la participación de la Universidad en la "Red Iberoamericana para la Aplicación de la Fotogrametría Arquitectural Digital y SIG para la Conservación del Patrimonio Histórico, Cultural y Arqueológico de Ciudades para una Gestión de Turismo Sustentable" (REFADC, 2019) promovida por CYTED.

En cuanto al Sistema de Información Geográfica, su desarrollo en la Universidad del Azuay se produce principalmente desde el año 1997 (Universidad del Azuay, 1997).

Los avances alcanzados a la fecha en los diversos ámbitos mencionados, se describen en el presente artículo.

\section{Metodología}

Los componentes que integran el trabajo desarrollado son:

- El Sistema de Información Geográfica

- El modelamiento fotogramétrico bidimensional (2D)

- El modelamiento fotogramétrico tridimensional (3D)

\section{EI Sistema de Información Geográfica}

Constituye el soporte operativo en el que se deposita la geoinformación tanto de los aspectos cartográficos, temáticos y digitales correspondientes a los modelos fotogramétricos generados. Se ha utilizado el software ArcGis en su versión 10.3.

En cuanto a la información con la que se ha generado el modelo, una parte corresponde a la utilizada en la Propuesta de Inscripción del Centro Histórico de Cuenca Ecuador en la lista de patrimonio mundial y que fuera aprobada por la UNESCO (UNESCO, 2019), la misma que corresponde a:

- Cartografía base: contratada por el municipio al Instituto Geográfico Militar del Ecuador (IGM), con el propósito de utilizarla en la actualización del catastro de la ciudad de Cuenca de 1997. Restituida a partir de fotografía aérea tomada en los meses de mayo y junio de 1994 a una escala 1:1000 y en formatos analógicos y digitales. 
- Catastro predial: con la información recogida mediante la ficha catastral que se usó en el proyecto de actualización catastral del año 1997 (Salgado-Arteaga \& Ochoa-Arias, 2011) en la que constan la información de todos los predios de la ciudad y particularmente las correspondientes al centro histórico.

- Modelo de base de datos y estructura gráfica: El modelo entidadrelación fue generado para recoger la información levantada en la ficha catastral dentro del proyecto "Modelo del Sistema de Información Geográfica de Cuenca" (Universidad del Azuay, 1997), el mismo que consta de 8 tablas relacionales vinculadas entre sí.

- Delimitación de las áreas históricas, según la ordenanza 28-04-1983 del municipio de Cuenca.

Otra parte de la información corresponde a:

- Caracterización de las edificaciones del área del Centro Histórico y el Ejido según el valor patrimonial de las mismas (Municipalidad de Cuenca, 2016).

- Identificación de las edificaciones que han modeladas digitalmente en sus fachadas, tanto bidimensionalmente como tridimensionalmente, así como sus respectivos vínculos web que las describen con detalle (IERSE, Universidad del Azuay, 2019)

- Ortofotografía de la zona de Cuenca, escala 1:5000 provenientes del Proyecto SIGTierras (MAG-SIGTierras, 2019)

Toda la información cartográfica originalmente utilizada para la preparación del expediente UNESCO, se migró desde el datum psad56 al wgs84, y con ello se logró volverla compatible con los otros mapas temáticos nuevos y las ortofotos de SIGTierras.

La información de la caracterización de las edificaciones según su valor patrimonial se generó digitalizando el mapa disponible en el portal de Cuenca Digital (Municipalidad de Cuenca, 2019)

\section{Modelamiento fotogramétrico bidimensional (2D)}

Para conseguir un modelo fotogramétrico digital de una edificación, corrigiendo la perspectiva natural con la que se visualiza el objeto desde un 
punto de observación, se aplica la técnica denominada fotogrametría (Jáuregui, 2008), la misma que consiste en proyectar el objeto sobre un plano de referencia de forma perpendicular al mismo (ortogonal), utilizando instrumentos (actualmente software) que procesan el relevamiento fotográfico obtenido de la edificación.

El procedimiento aplicado se denomina restitución digital, se lo puede realizar de forma automática o manual. Si se lo realiza de forma manual, se requiere que un operador, luego de ingresar al computador las fotografías del objeto, señale en la pantalla los puntos coincidentes en las diferentes fotografías, en cualquiera de los casos, al final se aplica un procedimiento de orientación en forma matemática en el que se correlacionan las imágenes para obtener el producto digital en formatos raster o vectorial.

\section{Relevamiento fotográfico}

Elegido el objeto a levantar (edificación), se procedió a la toma de fotografía en campo mediante una cámara digital Canon Rebel 3Ti 18mm. Para el efecto, la cámara fotográfica se ubica a una distancia conveniente del objeto, utilizando dos tipos de trípode, uno de altura corta (1.5 metros) y el otro larga (hasta 6 metros de altura).

$\mathrm{Al}$ inicio, se aplicó un enfoque automático del lente para conseguir un ajuste correcto, luego se pasa a modo manual y se fija la distancia focal (18 $\mathrm{mm}$ ) conforme la calibración aplicada por el software.

Se tuvo presente las recomendaciones que se ya han sido aplicadas (Ochoa-Arias, 2013):

- Captar las imágenes de manera que el ángulo formado entre las primeras y las últimas sea de 90 grados a manera de un semicírculo

- Procurar realizar la toma de imágenes en un día poco soleado, para evitar la inclusión de sombras que dificultarán el proceso de restitución que realice el computador.

- Utilizar en cada punto de toma al menos 3 fotografías, en una edificación simple se requieren 3 estaciones y en total al menos 10 fotografías

- Tomar con precisión al menos 2 medidas tomadas entre puntos o elementos a los que se tenga alcance, para incorporarlos al software y conseguir escalar el modelo. 


\section{Construcción del modelo}

El conjunto de fotografías obtenidas en campo, se cargaron al software Photomodeler 2012, eliminando previamente aquellas que son redundantes, tienen una calidad deficiente o el sistema no les reconoce como pertenecientes a la cámara utilizada.

Para la realización del procedimiento de orientación de las fotografías, se ubican los mismos puntos en varias de ellas, con el cual el software puede establecer los valores $\mathrm{x}, \mathrm{y}$ y $\mathrm{z}$ necesarios de cada punto y conseguir traslapar adecuadamente el mosaico fotográfico y establecer los ejes de coordenadas espaciales del conjunto.

A efecto de garantizar que el procedimiento funcione adecuadamente, se escogieron puntos que estén distribuidos de manera equilibrada, esto es, que cubran varios puntos geométricamente equidistantes en la edificación.

La tarea de construir el modelo bidimensional, requirió de realizar un trabajo interactivo en el que se busca alcanzar errores residuales menores a 5 pixeles, lo cual supone consultar constantemente la tabla de calidad de los puntos.

Los puntos se pueden observar en el espacio tridimensional y el sistema muestra la posición en la que se ubicó la cámara para cada estación de toma fotográfica, permitiendo detectar inconsistencias.

A este nivel de procesamiento es factible determinar la calidad del modelo que se está generando y si los procedimientos se realizaron adecuadamente.

A continuación, se procedió a señalar las áreas superficiales que constituyen las partes que componen el conjunto utilizando los puntos ya ubicados tridimensionalmente (Figura 2). Esta tarea permite corregir la visión en perspectiva que tiene las fotografías originales.

Con las superficies ya establecidas, se procede asignar las texturas, esto es el relleno fotográfico correspondiente a cada superficie. Este procedimiento lo puede realizar automáticamente el software, para lo cual escogerá del mosaico original, la porción fotográfica que mejor encaje en la misma.

Finalmente, el producto obtenido constituye la ortofoto de la edificación, es decir la fachada ortorrectificada en 2D (Figura 5a) 


\section{Figura}

\section{Asignación de texturas a las superficies obtenidas}

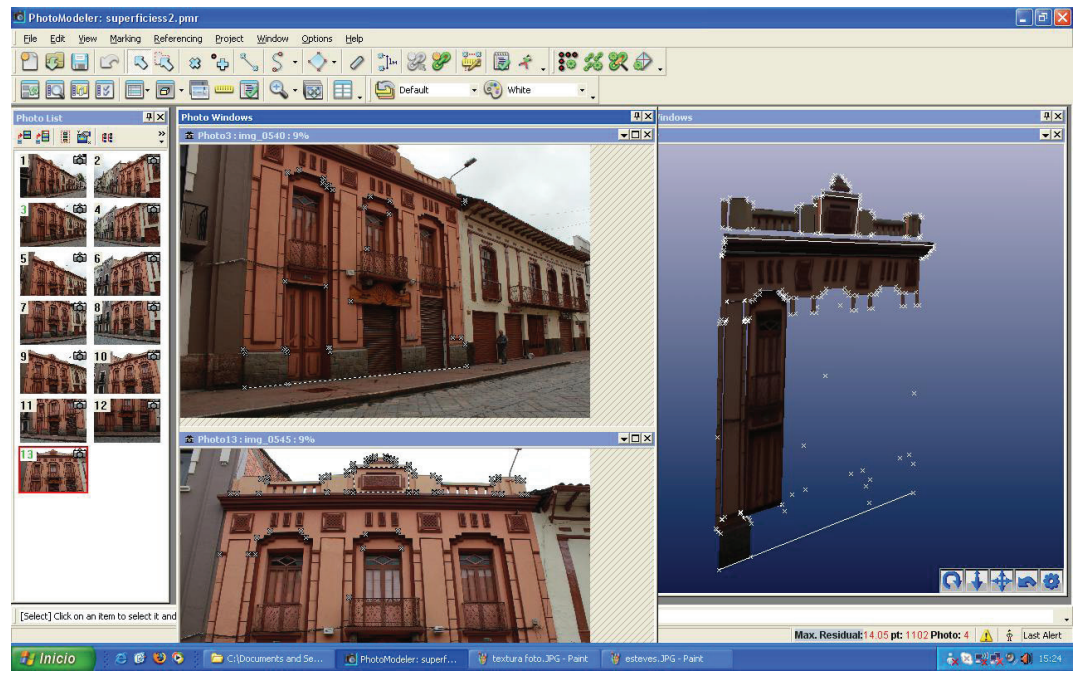

Fuente: Elaboración propia.

\section{Modelamiento fotogramétrico tridimensional (3D)}

Considerando el mismo principio utilizado para la ortogonalización de las fotografías que permiten obtener productos bidimensionales (2D), se genera el modelamiento tridimensional, pues resulta de la exportación del mismo producto a un formato habilitado para el procesamiento 3D.

El modelo generado se exporta a un formato vectorial que permite obtener el modelo 3D para posterior impresión en resina o filamento, para ello ha utilizado el siguiente equipo:

- Cámara digital Canon Rebel 3Ti 18mm

- Drone DJI Phantom 4 Pro

- Trípode

- Distanciómetro digital

- Flexómetro

- Programa informático Agisoft Metashape Pro

- Programa informático Meshlab y Meshmixer 
Para el levantamiento fotográfico, la cámara se ubica siguiendo las recomendaciones ya indicadas para el relevamiento fotográfico en 2D, la salvedad ocurre por las características del equipo utilizado, en este caso un dron, el mismo que requiere lineamientos específicos para su uso, de manera de facilitar el procesamiento de imágenes ya que el software Agisoft Metashape Pro, recomienda no cambiar la distancia focal pues podría ocasionar distorsiones de profundidad en los objetos a modelarse. Para el caso, se estableció una distancia focal de $18 \mathrm{~mm}$ en la cámara réflex y $8 \mathrm{~mm}$ en la cámara propia del dron.

Las previsiones que deben tomarse para el relevamiento fotográfico son las mismas que se indicaron en el acápite anterior, pero adicionalmente, cuando se trata del uso del dron, se preparó un plan de vuelo que asegura un barrido total de la edificación mediante una grilla ortogonal que, al ser recorrida por el aparato, tome imágenes cada 2 segundos.

En la primera secuencia de vuelo se captan imágenes de manera perpendicular a la fachada, haciendo un barrido horizontal a diferentes alturas (figura 3a). En la siguiente secuencia se recorre en sentido vertical desde el centro de la edificación mirando a los costados (figura 3b). Una última secuencia recorre en sentido vertical desde costados de la edificación mirando al centro (figura 3c) en sentido vertical mirando hacia los costados (figura 3c). Estas dos últimas tienen como propósito, captar las profundidades y detalles.

Para el modelamiento del objeto en 3D se desactiva del metadato de cada imagen, la asignación al sistema de coordenadas, con el propósito de evitar el escalamiento automático que hace el software, lo que limitaría las posibilidades de manipulación del modelo, luego de ello se llevan las fotografías al software Agisoft Metashape Pro.

Una vez ingresadas las imágenes se procedió a alinearlas, dentro de las configuraciones para la alineación de fotos que ofrece el software Metashape Pro seleccionamos la opción más alta (highest) pues interesa captar los detalles del objeto. A continuación, se procedió con la creación de la nube de puntos con nivel de detalle intermedio.

Finalmente se procede a generar la malla 3D, este paso es crucial ya que hasta este momento no se ha creado ningún objeto 3D implícito. Para la creación del mismo configuramos nuevamente las opciones del software en alta (high), este procesamiento hará que se genere una malla en base a la nube de puntos que anteriormente obtuvimos, triangulando la información de la misma para obtener una malla con información. 
Figura 3

Secuencias de trayectoria del dron para la captura de imágenes: a) barrido horizontal a diferentes alturas, b) barrido desde el centro con vistas a los costados, c) barrido desde los costados con vista al centro
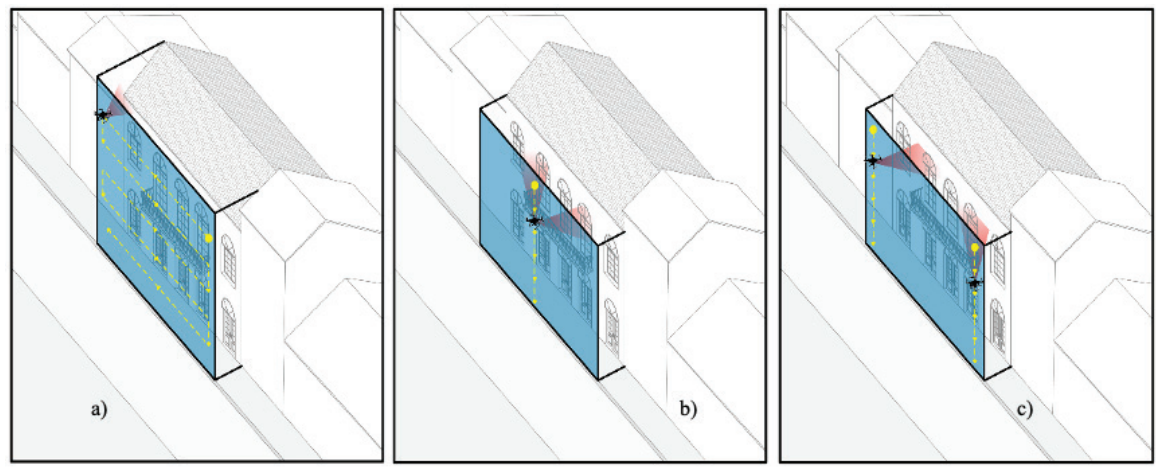

Fuente: Elaboración propia.

Una vez establecidas las superficies, se asignan las texturas mediante las opciones que ofrece Agisoft Metashape, esto es, se generará un mosaico que abarca las superficies creadas y a partir del mismo se consigue llevarlo a un formato de modelo digital de puntos TIN (Triangular Irregular Network) y a los que permiten las impresiones en 3D, tal como se observa en la figura 5, esto es, en el literal b) el modelo tridimensional con textura, en el c) el modelo tridimensional de puntos (TIN), y en el d) la impresión del modelo en 3D en mediante filamento plástico

\section{Resultados y discusión}

\section{El Sistema de Información Geográfica}

Se ha obtenido un modelo de gestión de información de edificaciones de valor patrimonial para el área de primer orden de la ciudad de Cuenca, el mismo que está soportado en un Sistema de Información Geográfica (figura 4), que cuenta con información predial (catastro), delimitación de las áreas 
de valor patrimonial, categorización de las edificaciones, identificación de edificaciones que cuentan con modelos digitales bidimensionales y tridimensionales, acceso a la información de la ficha catastral y al respectivo sitio Web que contiene información detallada del registro gráfico de la edificación (https://bit.ly/32inSVW).

Con respecto a la valoración del sistema generado, se la puede describir a partir de los aspectos relativos a su relevancia, efectividad, eficiencia y sostenibilidad.

- Relevancia: Las aplicaciones y simulaciones realizadas en ejercicios trabajados con el sistema madre, sobre el que se basa el desarrollo específico propuesto en este artículo, dan cuenta de su significación (Salgado-Arteaga \& Ochoa-Arias, 2011), tanto así que el sistema actual usado en el GAD Municipal de Cuenca (Municipalidad de Cuenca, 2019) está trabajado sobre el mismo modelo, pues tienen un origen común resultado del proyecto "Modelo del Sistema de Información Geográfica de Cuenca" (Universidad del Azuay, 1997).

- Efectividad: El origen común que tiene el sistema propuesto con el existente en la Municipalidad de Cuenca, hace que compartan información que asegura su interoperabilidad pues permite posibilidades de conexión a partir del código catastral y asegura su efectividad, pues establece un potencial de conexión con la información que disponga de la llave de acceso asignada, la misma que corresponde a la clave catastral.

- Eficiencia: Teniendo en cuenta que la información de naturaleza catastral la lleva el GAD Municipal de Cuenca, con la inherente responsabilidad de mantenerla actualizada, únicamente debe gestionares la capa temática de las Edificaciones Patrimoniales sin requerir de inversiones adicionales que supondría la gestión de todo el sistema.

- Sostenibilidad: El permitir la integración de la información con otras bases de datos, proyecta una posibilidad de continuidad pues se convierte en un engranaje más del sistema de información para la gestión territorial. A este nivel, conviene facilitar el establecimiento de un comité de gestión que señale las políticas generales y uno técnico que las opere (Universidad del Azuay, 1997), todo ello se podría compartir en una Infraestructura de Datos Espaciales (IDE) que podría tomar como nodo principal el implementado por el municipio de Cuenca. 
Hay que partir de la premisa que establece que, la gestión participativa del patrimonio cultural genera una dinámica que permita la sostenibilidad de las iniciativas, como lo expresan los trabajos de (Asmal- Guamán, 2019; Siguencia et al., 2018), dado que la concepción del objeto de trabajo incluye a la población, de manera que resulte en una mejora integral a partir de las potencialidades propias de cada comunidad como lo manifiesta Alfredo Conti (Conti, 2016), y sostenido por varios autores que resaltan la necesaria interacción entre el componente físico y humano de la ciudad (Carofilis \& García, 2015).

\section{Figura 4 \\ El sistema de Información Geográfica para la gestión de la información del patrimonio edificado}

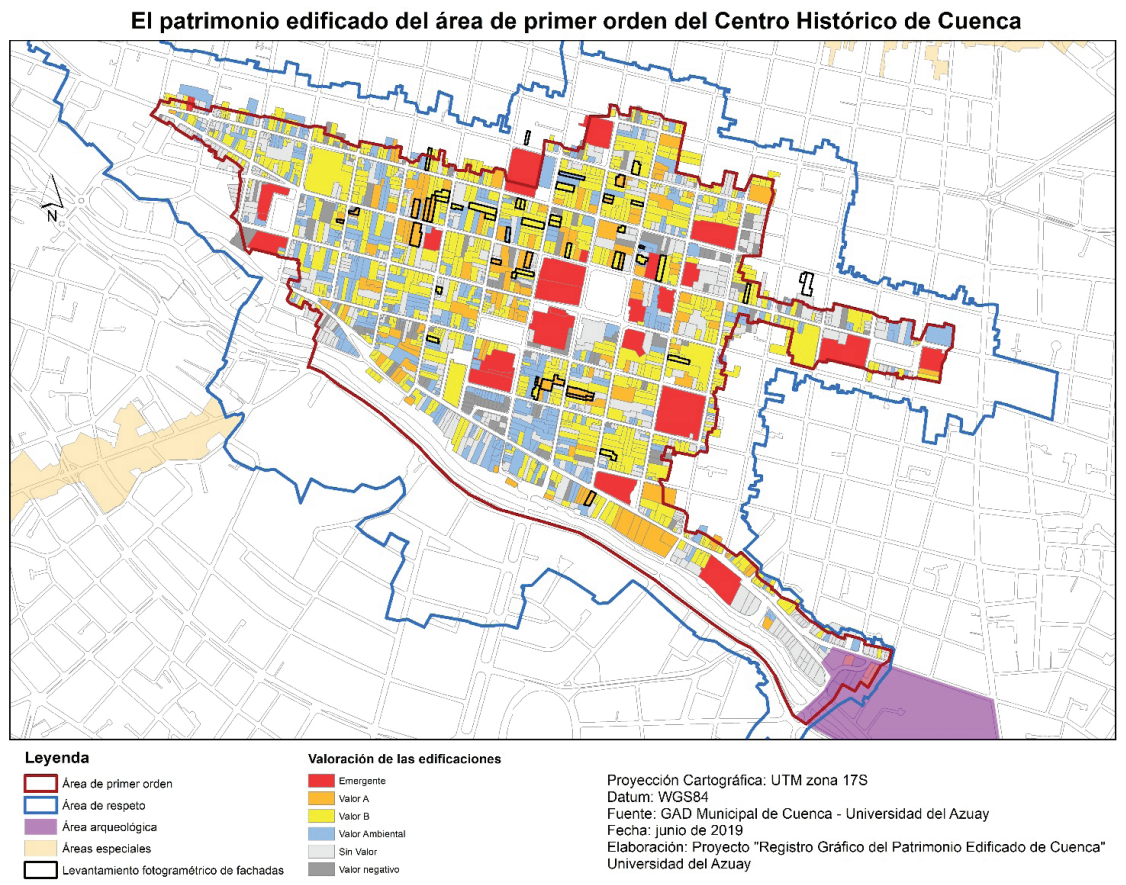

Fuente: Elaboración propia a partir de información del GAD Municipal de Cuenca. 
El impulso que el INPC (Instituto Nacional de Patrimonio Cultural) puede dar a la construcción del sistema es importante, pudiendo incorporar otros aspectos de naturaleza patrimonial como: arqueológico, documental, inmaterial y de bienes muebles, un ejemplo de ello lo constituye el proyecto "Aplicaciones de las Tecnologías de Información Geográfica (TIG) para la Gestión del patrimonio cultural en la provincia del Azuay" (López, 2009).

\section{Modelos fotogramétricos bidimensionales y tridimensionales}

Los modelos digitales obtenidos han requerido de un proceso reiterativo de depuración que permite registrar con fidelidad al objeto real, la dificultad surge de la necesidad de captar la mayor cantidad de imágenes fotográficas desde todos los ángulos posibles en procura de que los detalles queden registrados adecuadamente, particularmente cuando se pretende obtener un modelo tridimensional, esto implica ubicar la cámara fotográfica a diferentes alturas, posiciones y lo más cercana al objeto, lo cual ha sido posible únicamente con el uso del dron, algunos estudios comparativos entre diferentes técnicas posibles de aplicar dan cuenta de sus ventajas (Preti \& Tituana, 2017) y señalan su bajo costo (Pacheco-Prado, 2017).

Los productos obtenidos son: 50 fachadas ortorrectificadas en 2D, 5 fachadas ortorrectificadas en 3D, generadas en realidad virtual e impresas en filamento plástico, así como varios tramos de la ciudad levantadas para análisis de concordancia estética. Estos productos se pueden descargar o visualizar en el sitio web http://gis.uazuay.edu.ec/patrimonio.php. En la Figura 5 , se observan un ejemplo de los productos indicados. Para la fachada de la edificación signada con el número 28 (código interno del levantamiento), correspondiente a la calle Bolívar 13-89 de la ciudad de Cuenca, se observan en dicha figura: la fachada ortorrectificada en 2D (a), fachada ortorrectificada en 3D (b), el modelo tridimensional de puntos (TIN) (c) y la impresión 3D en filamento (d). 


\section{Figura 5}

Productos obtenidos: a) fachada ortorrectificada en 2D, b) modelo tridimensional con textura, c) modelo tridimensional de puntos (TIN), d) impresión del modelo en 3D en mediante filamento plástico

a)

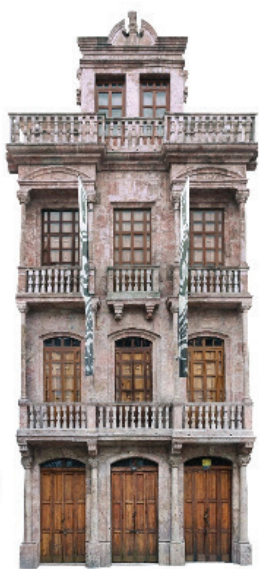

b)

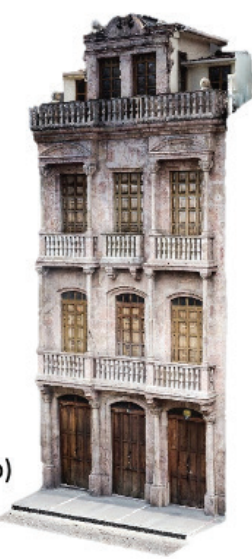

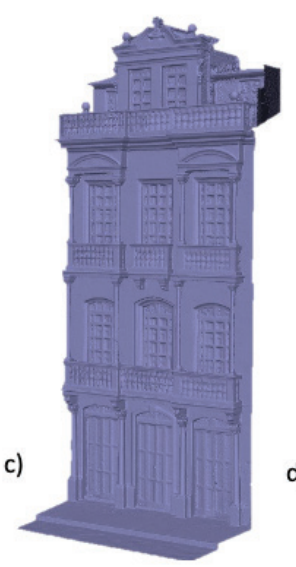

Fuente: Elaboración propia.

\section{Conclusión}

Las disponibilidades técnicas y metodológicas han permitido generar un modelo de información documentada digitalmente e integrada en un Sistema de Información Geográfica; en el cual dispone de información predial (ficha catastral), delimitación de las áreas de valor patrimonial, representación de las edificaciones bidimensional y tridimensionalmente, acceso al respectivo sitio Web, y un potencial amplio para la gestión del del patrimonio edificado.

La conveniencia de generar una gestión participativa del patrimonio, hace necesario realizar un mayor esfuerzo para asegurar la integración de organismos y de la información que les compete. La existencia de una creciente disponibilidad de procedimientos, técnicas, instrumentos y sobre todo 
de conocimientos, como se ha señalado en el presente artículo, permitirían la gestión patrimonial en una modalidad concurrente y compartida.

Se requiere, sin embargo, la detonación de una decisión administrativa dirigida a concretar el surgimiento de estos espacios. Para ello se podría propiciar que uno de los actores tome la iniciativa, sin embargo, el sistema debería ser administrado por los organismos locales de carácter público.

No debe concentrarse físicamente la información, además debe compartirse el uso de los datos y el conocimiento necesario para usar la tecnología que los gestiona, esto último supone que las universidades dinamicen el correspondiente componente de investigación y capacitación.

Sería deseable generar una IDE (Infraestructura de Datos Espaciales), para lo que resulta conveniente desarrollar un modelo del mismo, en el que se podría plantear una distribución de responsabilidades y permitir la confluencia de la visualización de los productos generados por las diferentes fuentes o nodos en los que residiría.

El modelo de la IDE Patrimonial, debería surgir de partida asegurando su interoperabilidad con las IDE ya existentes, que tienen cierto grado de consolidación, como, por ejemplo: "La plataforma de información para la planificación territorial de la Zona 6" del Ecuador.

\section{Bibliografía}

Asmal-Guamán, D. (18 de junio de 2019). Modelo de gestión del patrimonio cultural edificado basado en la participación ciudadana para la ciudad de Cuenca. http://bit.ly/37w2CgN

Carofilis, N., \& García, G. (2015). El patrimonio como recurso: el cambio de paradigmas en la conservación urbana desde una perspectiva internacional. ESTOA(6), 69-80. https://doi.org/10.18537/est.v004.n006.12

Conti, A. (2016). Paisajes históricos urbanos: nuevos paradigmas en conservación urbana. MEC-EDUPAZ, 2(10), 1-12. https://bit.ly/3grcAnh

IERSE, Universidad del Azuay (14 de junio de 2019). El patrimonio Edificado de Cuenca. https://bit.ly/3aTD8wf

Jáuregui, L. (2008). Introducción a la Fotogrametría. Universidad de los Andes.

López, F. (2009). Aplicaciones de las Tecnologías de Información Geográfica (TIG) para la Gestión del patrimonio cultural en la provincia del Azuay. Universidad Verdad, (49), 123-166. 
MAG-SIGTierras (14 de junio de 2019). Geoportal SIGTierras. https://bit.ly/3jdyKv5 Municipalidad de Cuenca (2016). Plan Especial del Centro Histórico. Cuenca.

Municipalidad de Cuenca (14 de junio de 2019). http://bit.ly/38MRSun

Ochoa-Arias, P. (2013). Fotogrametría Arquitectural: una herramienta para la Gestión del Patrimonio Edificado. Universidad Verdad (60), 263-292.

Pacheco-Prado, D. (2017). Drones en espacios urbanos: Caso de estudio en parques, jardines y patrimonio edificado de Cuenca. Estoa, Revista de la Facultad de Arquitectura y Urbanismo de la Universidad de Cuenca, 6(11), 159168. http://dx.doi.org/10.18537/est.v006.n011.a12

Pisón, E.M. (1998). Paisaje y medio ambiente. Seminario Paisaje y Medio Ambiente. Universidad de Valladolid, Secretariado de Publicaciones e Intercambio Editorial.

Preti, P., \& Tituana, K. (21 de junio de 2017). Metodología de documentación digital del patrimonio aplicado a la arquitectura con características modernas en la ciudad de Cuenca. http://bit.ly/2U0VeWw

REFADC (12 de junio de 2019). Red Iberoamericana para la aplicación de la Fotogrametría Arquitectural Digital y SIG para la conservación del Patrimonio Histórico, Cultural y Arqueológico de ciudades para una gestión de turismo sustentable. https://bit.ly/34rwZpJ

Roura, A., \& Ochoa, P. (2014). Influencias de estilos arquitectónicos en el Centro Histórico de Cuenca. Universidad Verdad,64, 99-118. https://doi. org/10.33324/uv.v0i64.256

Salgado-Arteaga, F., \& Ochoa-Arias, P. (2011). Modelo del Sistema de Información Geográfica de Cuenca: validación y actualización transcurridos 15 años de su creación. En F. Salgado Arteaga, Ingeniería y Territorio (p. 168). Cuenca.

Santana, M. (2013). Antecedentes, rol y desafío de la digitalización del patrimonio arquitectónico. Estoa, 3, 7-21. https://doi.org/10.18537/est.v002.n003.02

Siguencia, M., Vintimilla, S., \& Auquilla, S. (2018). El paisaje en la perspectiva de la comunidad, identificando Patrimonio en Cuenca-Ecuador. ASRI(14), 236-253. https://bit.ly/3j9fajn

UNESCO (11 de junio de 2019). Historic Centre of Santa Ana de los Ríos de Cuenca. https://bit.ly/31oNbGD

Universidad de Cuenca/GAD Municipal del Cantón Cuenca (2017). Propuesta de Inscripción del Centro Histórico de Cuenca Ecuador en la lista de patrimonio mundial. Edición Comentada 2017. Cuenca: Universidad de Cuenca. 
Universidad del Azuay (1997). Modelo del Sistema de Información Geográfica de Cuenca. (U. d. Azuay, Ed.) Cuenca, Ecuador: Universidad del Azuay.

Fecha de envío: 2020/01/27; Fecha de aceptación: 2020/08/23;

Fecha de publicación: 2020/09/01 